Check for updates

Cite this: RSC Adv., 2017, 7, 43879

Received 13th July 2017

Accepted 31st August 2017

DOI: 10.1039/c7ra07694k

rsc.li/rsc-advances

\section{Low resistance bicomponent spunbond materials for fresh air filtration with ultra-high dust holding capacity $\dagger$}

\author{
Jinxin Liu, (D) Xing Zhang, Haifeng Zhang, Lei Zheng, Chen Huang, (DD Haibo Wu, \\ Rongwu Wang and Xiangyu Jin*
}

In fresh air ventilation systems, filters with high dust holding capacity and low resistance are required to prolong their service life and reduce energy consumption. However, with the current filtration materials it is difficult to achieve this due to the limitation of the packing density and fiber diameter. Herein, a polypropylene/polyethylene (PP/PE) core/sheath bicomponent spunbond technique is proposed to fabricate airborne particle filtration materials with a three-dimensional (3D) fluffy structure. The properties of the bicomponent spunbond (BCS) materials, including fiber diameter, morphology, tensile strength, pore structure, and porosity, can be finely controlled by regulating the processing parameters such as the quenching air temperature, drawing air pressure, and bonding method. The resulting BCS materials exhibit a low pressure drop of $35.14 \pm 2.01 \mathrm{~Pa}$, an ultrahigh dust holding capacity of $9.36 \pm 0.52 \mathrm{~g} \mathrm{~m}^{-2}$, and a relatively high filtration efficiency of $97.02 \% \pm 0.8 \%$. The successful fabrication of such intriguing materials could provide a new approach for the design and development of fresh air filtration materials.

\section{Introduction}

Particulate matter (PM) pollution, especially that caused by PM 2.5 (aerodynamic diameters less than $2.5 \mu \mathrm{m}$ ) and PM 10 (aerodynamic diameters from 2.5 to $10 \mu \mathrm{m}$ ), is becoming increasingly serious in developing countries such as China and India, which exerts severe impacts on people's living environment, such as on the air visibility or air quality, and thus people's health. ${ }^{1-3}$ Controlling and reducing the pollution sources is essential to solve the problem, which is a long-term process related to making policies and upgrading industries. ${ }^{4}$ Therefore, utilizing fresh air ventilation systems to filter air before breathing is now the most effective way to protect people from the influences of air pollution., ${ }^{5,6}$

To date, porous membrane materials and fibrous materials are the two major classes of air filtration media. The particle capture is mainly based on two major deposition patterns (surface filtration and deep deb filtration) and five main filtration mechanism including physical sieving, inertial separation, interception, diffusion, and electrostatic attraction..$^{7-9}$ Conventional filtration materials usually rely on physical sieving. Consequently, the pore size of the filtration materials has to be reduced to achieve higher filtration efficiency, resulting in super

Engineering Research Center of Technical Textiles, Ministry of Education, College of Textiles, Donghua University, No. 2999 North Renmin Road, Songjiang, Shanghai 201620, China. E-mail: jinxy@dhu.edu.cn

$\dagger$ Electronic supplementary information (ESI) available. See DOI: 10.1039/c7ra07694k high pressure drop and thus excessive energy consumption and noise pollution..$^{10}$ Nowadays, corona charging treatment has been widely used to improve the filtration efficiency of fibrous materials without increasing the pressure drop by making full use of electrostatic attraction. ${ }^{11,12}$ Although improved filtration performance of the electret melt-blown nonwovens and electrospun materials have been observed, their air resistance is pretty high. In addition, spunbond materials own lower air resistance and better mechanical properties by comparison. Made of synthetic polymers, spunbond materials were commercialized by Freudenberg (Germany) and Du Pont (USA) between the 1950's and 1960's. ${ }^{13}$ Nonetheless, traditional spunbond materials usually adopt calender bonding reinforcement, resulting in high air resistance due to high packing and low dust holding capacity.

Bicomponent spunbond materials are promising to produce materials having a fluffy structure by using through-air bonding reinforcement. ${ }^{14}$ The cross-section of bicomponent fibers produced by bicomponent spunbond technique varies from islands-in-the-sea, ${ }^{15}$ hollow segmented-pie, ${ }^{16}$ side-by-side, ${ }^{17}$ to core/sheath, etc. ${ }^{18}$ Among them, the polypropylene/polyethylene ( $\mathrm{PP} / \mathrm{PE})$ core/sheath bicomponent spunbond (BCS) materials can be made into diverse cavity structures under different thermal bonding conditions.

In this study, fluffy electret fibrous materials (FEFM) with micron fiber size were fabricated in order to achieve the effect of low resistance and high dust holding capacity, by combining the bicomponent spunbond and corona charging techniques. It should be noted that the bicomponent spunbond technique 
was employed to generate materials with a fluffy structure to reduce air resistance and increase dust holding capacity, and the corona charging treatment was used to improve the filtration efficiency. Particularly, the effects of processing parameters on tensile strength, pore structure, and porosity of PP/PE BCS materials were investigated. Benefiting from the enhanced properties, the spunbond materials with high porosity and three-dimensional fluffy structure exhibit low resistance and high dust holding capacity, which could be a good candidate for the fresh air filtration system.

\section{Experimental}

\section{Materials}

PP pellets with a melt flow index (MFI) of $30 \mathrm{~g}(10 \mathrm{~min})^{-1}$ and PE pellets with a MFI of $20 \mathrm{~g}(10 \mathrm{~min})^{-1}$ were kindly provided by Sinopec Zhenhai Refining \& Chemical Company (Ningbo, Zhejiang, China) and PetroChina Co., Ltd. (Fushun, Liaoning, China), respectively.

\section{Fabrication of PE/PP BCS materials}

A 50 centimeter-wide bicomponent spunbond research line, located at the Nonwoven Research and Development Center of Donghua University (Shanghai, China) was used to produce the $\mathrm{PP} / \mathrm{PE}(1: 1, \mathrm{v} / \mathrm{v})$ core/sheath spunbond materials. Unless otherwise noted, the base weight of the spunbond materials was $120 \mathrm{~g} \mathrm{~m}^{-2}$.

\section{Corona charging}

In this study, the corona charging apparatus consists of a high voltage power supplier (DW-P503-2AODE, Tianjin Dongwen High-Voltage Power Supply Co., Ltd., Tianjin, China), an array of needles, and a copper holder (Fig. S1 $\dagger$ ). Before corona charging, a PP/PE spunbond sample was placed on a copper holder. After applying high potential between the needles and the copper holder, ions were generated and deposited on the PP/PE spunbond sample when corona discharge took place. In this study, all the prepared materials were corona charged before testing filtration performance. The applied voltage, the charging distance between the needles and the copper holder, and the charging time were $100 \mathrm{kV}, 100 \mathrm{~mm}$ and $30 \mathrm{~s}$, respectively.

\section{Characterization}

A Scanning Electron Microscope (SEM; TM3000, Hitachi Ltd., Japan) was used to examine the morphology of the spunbond materials. The specimens were sputter-coated with gold in order to avoid charge accumulations before observation. The fiber diameter was determined by measuring 100 fibers according to the SEM images, with the help of the software Nano Measurer 1.2.5.

Fourier Transform Infrared (FTIR) analyses were carried out by using a spectrometer (6700, Thermo Fisher Nicolet Inc., USA). The test was performed at room temperature and the spectra were recorded in a range from 4000 to $600 \mathrm{~cm}^{-1}$, with a resolution of $4 \mathrm{~cm}^{-1}$.
The pore size and distribution of spunbond materials were characterized through a bubble-point test using a capillary flow porometer (CFP-1100AI, Porous Materials Inc., USA).

A tensile tester (YG026MB-250, Wenzhou Fang Yuan Instrument Co. Ltd., Wenzhou, Zhejiang, China) was used to test the mechanical properties of the spunbond materials. The size of samples, tensile speed, clamp distance, and pre-applied force were $200 \times 50 \mathrm{~mm}^{2}, 200 \mathrm{~mm} \mathrm{~min}^{-1}, 100 \mathrm{~mm}$, and $2 \mathrm{~N}$, respectively.

Filtration performance was measured by an automated filter tester (TSI 8130, TSI lnc., USA), which could generate sodium chloride aerosol with different flow rate. The mass mean diameter of aerosol particles was $0.26 \mu \mathrm{m}$, and the geometric standard deviation of the particles was less than 1.83. Chargeneutralized aerosol particles were delivered through the spunbond nonwovens, which were clamped by a filter holder with an effective area of $100 \mathrm{~cm}^{2}$. An electron-laser particle photometer was used to measure the concentration of the aerosol particles in the upstream $\left(C_{\mathrm{u}}\right)$ and downstream $\left(C_{\mathrm{d}}\right)$ respectively. Filtration efficiency $\eta$ was calculated as follows: $\eta=\left(1-C_{\mathrm{d}} / C_{\mathrm{u}}\right) \times$ $100 \%$. The filtration performance of the materials was tested under the industrial standard face velocity of $32 \mathrm{~L} \mathrm{~min}^{-1}$. We weighed the samples before and after loading filtration when the load resistance reached $1000 \mathrm{~Pa}$ at high airflow speed of $85 \mathrm{~L} \mathrm{~min}^{-1}$, and the weight disparity represented the volume of the dust holding capacity.

\section{Statistical analysis}

At least three replicates were performed on each sample, and the values were expressed as average \pm standard deviation. Oneway analysis of variance was adopted, and $\alpha$-value was set at 0.05 . If $F>F$-crit, the differences among data were considered statistically significant, and were marked by *.

\section{Results and discussion}

In this study, $\mathrm{PP} / \mathrm{PE}$ core/sheath spunbond materials were produced by bicomponent spunbond technique (Fig. 1) and further treated with corona charging to improve the filtration

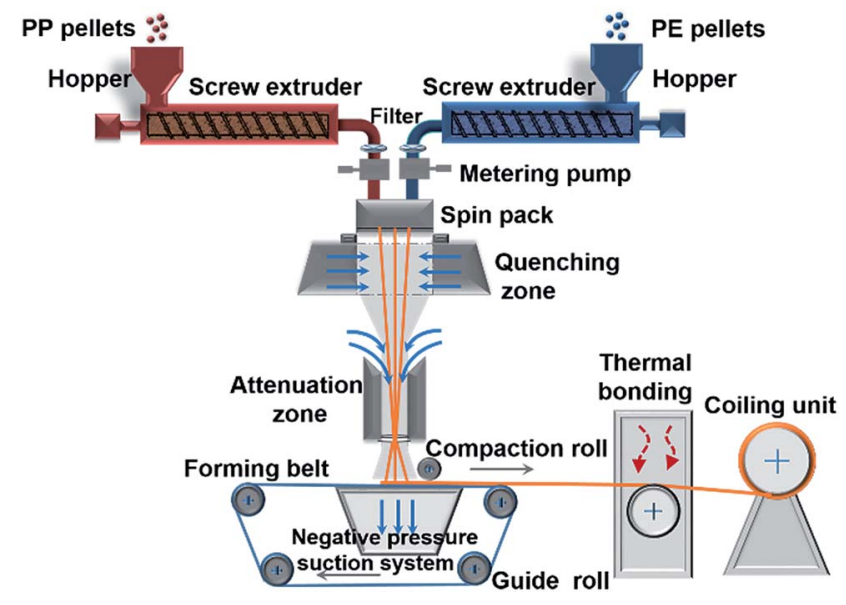

Fig. 1 Schematic diagram of the bicomponent spunbond process. 
performance (Fig. S1†). In brief, PP and PE pellets were firstly fed through two separate raw material hoppers to two screw extruders to heat and melt. Then the melt polymers entered the die through two separate melt filters and metering pumps, and then reached the spin pack after the even distribution. The melted PP and PE polymers were combined at the outlet of the spin pack, forming melt core/sheath bicomponent jets. The melt jets were further cooled and stretched by air to be finer and longer in the quenching zone and attenuation zone. Finally, spunbond materials were formed after collecting and the thermal bonding process. In summary, the air temperature in the quenching zone, air pressure in the attenuation zone, and the thermal bonding process are the main parameters in the spunbond process.

The FTIR spectrum of fiber surface showed that the characteristic curve corresponded to PE standard infrared spectroscopy (Fig. S2a $\dagger$ ), indicating the sheath component was PE exclusively. Then we sheared the fibers, ground them into powder and tested them again. The peak observed at $1376 \mathrm{~cm}^{-1}$ was attributed to the $-\mathrm{CH}_{3}$ symmetrical deformation vibration
(Fig. S2 $\mathrm{b}_{\dagger} \dagger$ ), indicating the existence of PP component inside the fibers. The comparison between the results of FTIR and the images obtained by SEM (Fig. 2a) confirmed the successful fabrication of $\mathrm{PP} / \mathrm{PE}$ core/sheath spunbond materials.

\section{Effect of the air temperature in the quenching zone on the properties of spunbond materials}

The cooling conditions in the quenching zone are the most vital in the spunbond process, which include the quenching air temperature, the quenching air speed, and the length of quenching zone. The effect of quenching air temperature on the properties of nonwovens was analysed by keeping the quenching air speed and the length of quenching zone constant at $1.2 \mathrm{~m} \mathrm{~s}^{-1}$ and $0.6 \mathrm{~m}$, respectively. Meanwhile, the drawing air pressure in the attenuation zone was $160 \mathrm{kPa}$, and calender bonding was adopted, the temperatures of upper and lower roller were 134 and $133{ }^{\circ} \mathrm{C}$, respectively.

The representative SEM images indicated that the fibers in the spunbond materials were randomly oriented when the
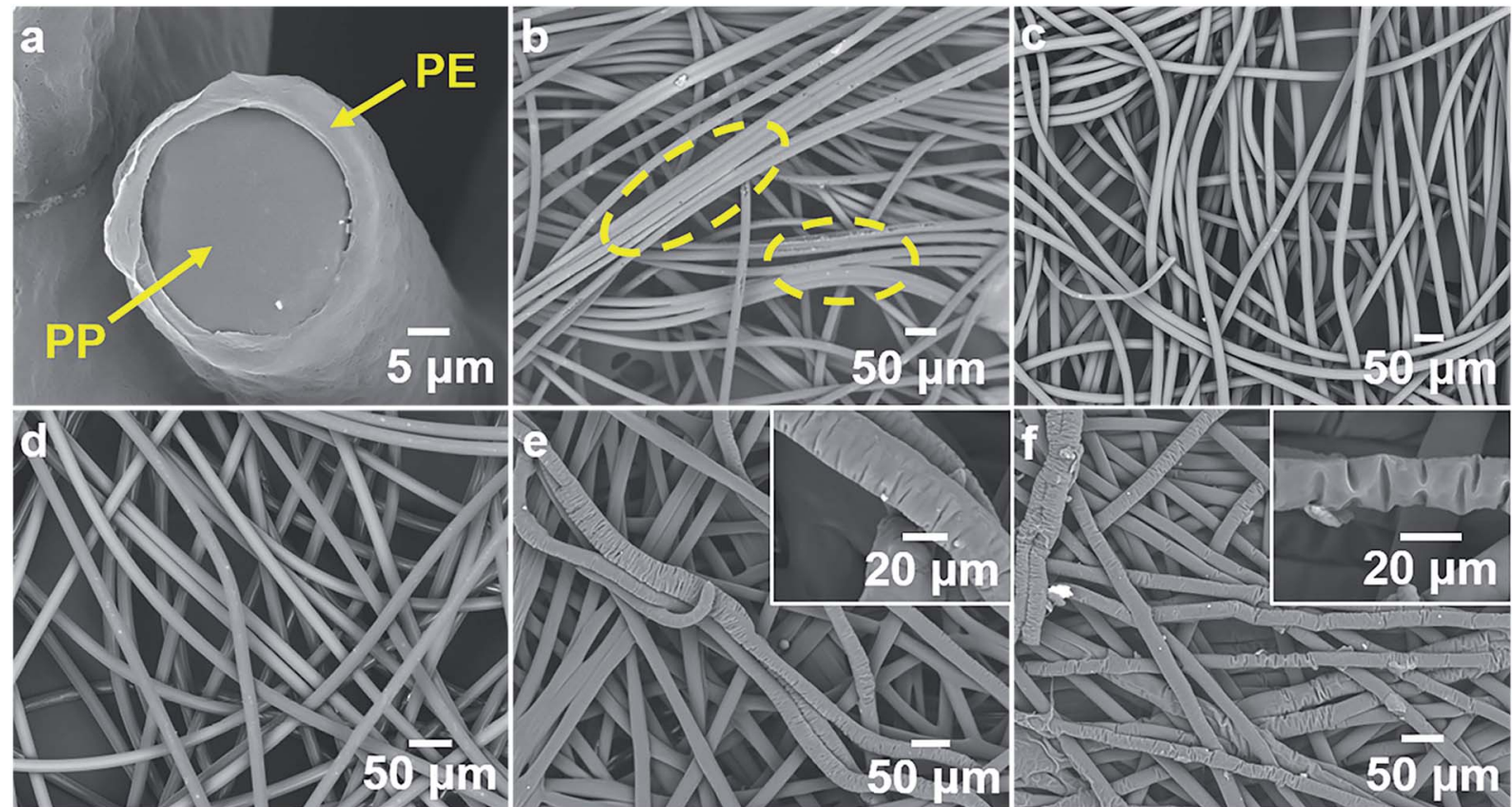
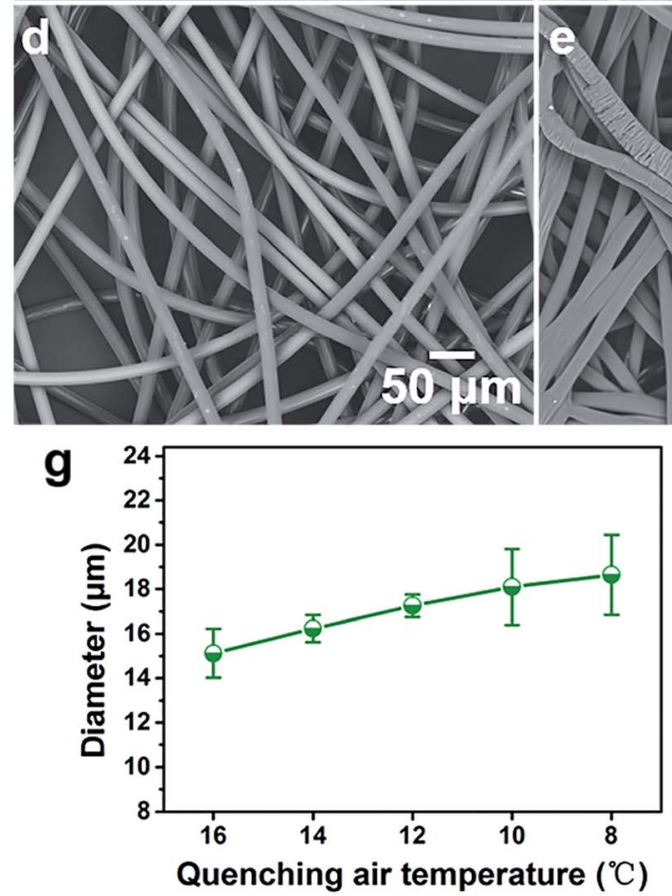

h

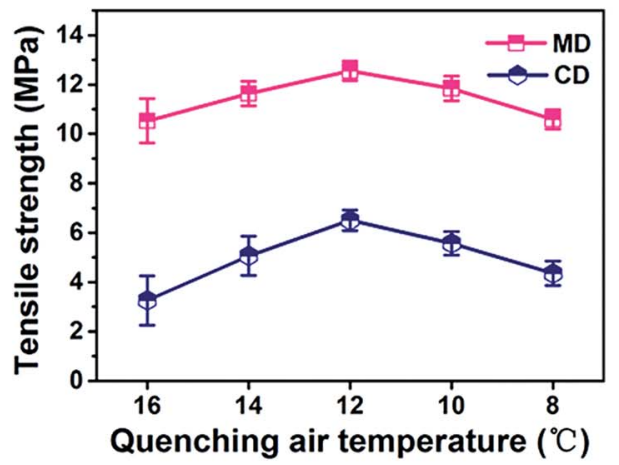

Fig. 2 Morphology, diameter, and mechanical property of PE/PP BCS materials. SEM images of (a) the cross section of the PE/PP bicomponent fiber, and webs obtained with various quenching air temperatures of (b) $16^{\circ} \mathrm{C}$, (c) $14^{\circ} \mathrm{C}$, (d) $12{ }^{\circ} \mathrm{C}$, (e) $10^{\circ} \mathrm{C}$, (f) $8{ }^{\circ} \mathrm{C}$. (g) Fiber diameter, (h) tensile strength of PE/PP BCS materials fabricated under various quenching air temperatures. 
quenching air temperature was varied (Fig. 2b-f). It should be noted paratactic fibers with a smooth surface were observed when the quenching air temperature was $16{ }^{\circ} \mathrm{C}$ (Fig. 2b). The doubling phenomenon should be ascribed to high temperature as the fibers couldn't be timely cooled down. By decreasing the quenching air temperature to $14{ }^{\circ} \mathrm{C}$ and $12{ }^{\circ} \mathrm{C}$, the paratactic fibers were rarely found, and the fibers turned to be more random (Fig. 2c and d). When the quenching air temperature was further lowered to $10^{\circ} \mathrm{C}$ and $8{ }^{\circ} \mathrm{C}$, a large number of distinct wrinkles formed on the surface of the fibers (Fig. 2e and f). The formation of these wrinkles should be attributed to the uneven cooling speed of the inside/outside the bicomponent fibers since the quenching air temperature was too low. Slower cooling speed of the inside of fibers led to the incomplete solidification, while the outside was shrunken during the spinning, thus the fiber surface became wrinkled.

In addition, the diameter of the spunbond fibers was enhanced from $15.12 \pm 1.1$ to $18.64 \pm 1.8 \mu \mathrm{m}$ when the cooling air temperature was lowered from 16 to $8{ }^{\circ} \mathrm{C}$ (Fig. $2 \mathrm{~g}$ ), indicating that higher quenching temperature could facilitate the fabrication of finer fibers due to lower solidification speed at higher temperature and thus more adequate stretching. However, the spunbond materials both in machine direction (MD) and cross direction (CD) exhibited the highest tensile strengths of $12.56 \pm$ 0.39 and $6.51 \pm 0.42 \mathrm{MPa}$, respectively, at the cooling air temperature of $12{ }^{\circ} \mathrm{C}$ (Fig. 2h). This is may be due to the fact that when the temperature was lower or higher, there would be more defects in the phenomena of wrinkling or doubling.

\section{Effect of the drawing air pressure in the attenuation zone on the property of PE/PP BCS materials}

Using the optimized cooling air temperature of $12{ }^{\circ} \mathrm{C}$, we next investigated the effect of the drawing air pressure in the attenuation zone on the morphology of the obtained spunbond materials (Fig. 3a-d). It was found that all the fibers showed smooth surface without doubling phenomenon and the fiber diameter decreased significantly from $18.98 \pm 0.82$ to $13.23 \pm$ $0.45 \mu \mathrm{m}$ when the drawing air pressure was increased from 140 to $200 \mathrm{kPa}$ (Fig. 3e), indicating that finer fibers were produced at higher air pressure attributing to larger air stretching force. ${ }^{19,20}$ It should be noted that the fiber diameter of spunbond materials is larger than that of meltblown and electrospun fibers (Fig. S3†), which is still suitable as low resistance air filtration materials.

Pore size and pore size distribution of BCS materials, directly related to fiber diameter, were measured through a capillary flow porometer. The materials with drawing air pressure of 140 , 160,180 , and $200 \mathrm{kPa}$ showed the pore size distribution in the range of 9-53 $\mu \mathrm{m}$, with well-developed peaks centered at 28.6 , $26.3,19.8$, and $15.1 \mu \mathrm{m}$, respectively. Moreover, the pore distribution exhibited a narrower trend when the drawing air pressure increased from 140 to $200 \mathrm{kPa}$ (Fig. 3f).

The filtration performance of the BCS materials was systematically investigated by using the charge neutralized sodium chloride $(\mathrm{NaCl})$ particles. Due to the fact that small airborne particles are difficult to control artificially, let alone to control them to flow at different rates, thus $\mathrm{NaCl}$ aerosol particles are generally used in the filtration industry as well as scientific research. ${ }^{21-23}$ The filtration efficiencies of the BCS materials formed with the air drawing pressures of 140, 160, 180 , and $200 \mathrm{kPa}$ were $65.81 \% \pm 1.8 \%, 75.84 \% \pm 1 \%, 85.6 \% \pm$ $1.4 \%$, and $92.32 \% \pm 0.5 \%$, while corresponding pressure drops were $35.42 \pm 4.5,52.97 \pm 5.1,65.85 \pm 3.4$, and $81.34 \pm 4.5 \mathrm{~Pa}$, respectively. That revealed an obvious enhanced trend of the filtration efficiency, as well as a significantly increased trend of the pressure drop (Fig. 4a). The improved filtration efficiency
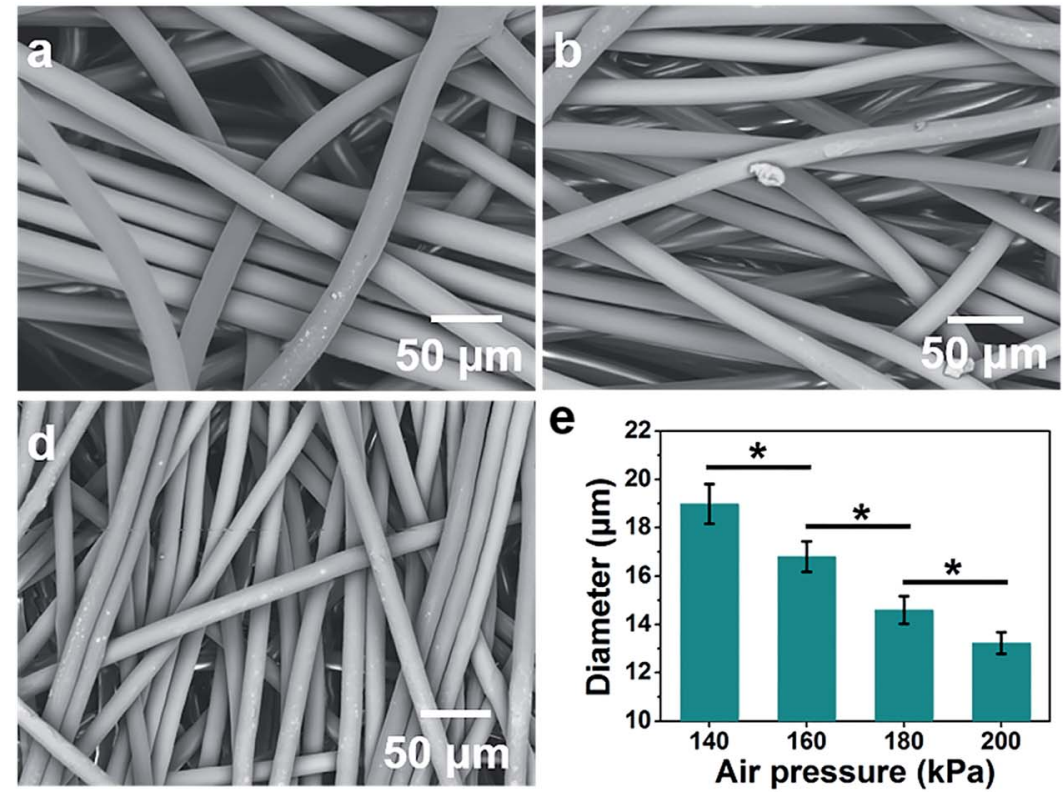
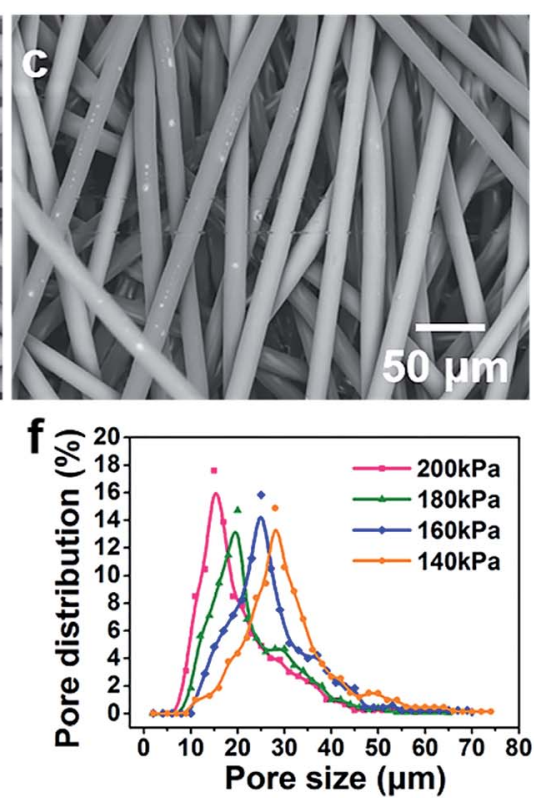

Fig. 3 SEM images of PE/PP BCS materials formed with various drawing air pressures (a) $140 \mathrm{kPa}$, (b) $160 \mathrm{kPa}$, (c) $180 \mathrm{kPa}$, (d) $200 \mathrm{kPa}$ under quenching air temperature of $12{ }^{\circ} \mathrm{C}$. (e) Fiber diameter and (f) pore size and pore size distribution curves. 

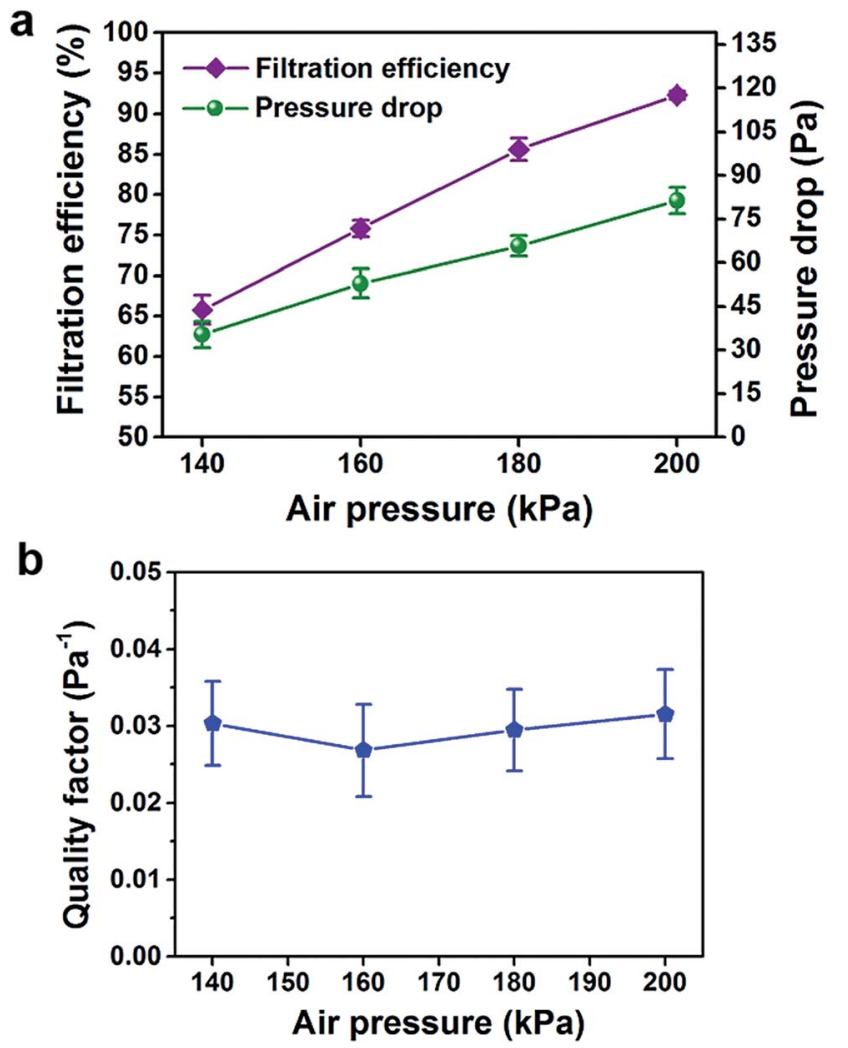

Fig. 4 (a) Filtration efficiency and pressure drop, and (b) quality factor of bicomponent materials fabricated from various air pressures under a face velocity of $32 \mathrm{~L} \mathrm{~min}^{-1}$.

and pressure drop could be attributed to the gradually decreased pore size, which was caused by higher drawing air pressure. To comprehensively evaluate the filtration performance of a given filtration medium, the trade-off parameter of quality factor $(\mathrm{QF})$ was introduced, ${ }^{22,24}$ and it could be calculated by the following formula:

$$
\mathrm{QF}=-\frac{\ln (1-\eta)}{\Delta p}
$$

$\eta$ and $\Delta p$ represented the filtration efficiency and pressure drop, respectively. This equation clearly indicated that large QF values required high filtration efficiency and/or low pressure drop, and a large quality factor indicated good filtration performance. $^{25}$ The QF value decreased first and then increased to the maximum value of $0.03152 \pm 0.0058 \mathrm{~Pa}^{-1}$ (Fig. 4b). Smaller fiber diameter and pore size were conductive to the filtration efficiency, and it seemed that we should pursue higher air pressure to make it. However, when air pressure exceeded $200 \mathrm{kPa}$, the spinning became very unstable and appeared filament break, which made the production fail to be carried out smoothly.

\section{Thermal bonding reinforcement selection}

According to previous reports on spunbond materials, the structure and morphology of webs are strongly affected by reinforcement process. ${ }^{\mathbf{1 3 , 2 6}}$ We further explored the influence of thermal bonding reinforcement on the spunbond materials when the quenching air temperature was $12{ }^{\circ} \mathrm{C}$ and the drawing air pressure was $200 \mathrm{kPa}$. Calender bonding and through-air bonding are two commonly used methods of thermal bonding reinforcement. In this study, we analyzed the effects of the thermal bonding reinforcement on the morphology, filtration performance, porosity, and dust holding capacity of the obtained BCS materials.

In calender bonding process, the web was passed through the nip of two rolls. Regarding the two rolls pressed against each other, one was the engraved roll and the other was smooth roll (Fig. 5a), both of which were heated internally. The temperatures of the upper and lower roller were 134 and $133{ }^{\circ} \mathrm{C}$, respectively. The heat flows were mainly through conduction into selection areas of the web; these areas, i.e. the bonding sites, were arranged in a uniform and repetitive pattern (Fig. 5b and c). While in the through-air bonding process, the heated air $\left(\sim 135{ }^{\circ} \mathrm{C}\right)$ went through the fiber web, so that the sheath component of PE melted and bonded; the core component of PP did melt and maintain stable structure. Suction boxes located inside the drum facilitated the heated air's passage through the web. Under those circumstances, every fiber crossover point in the web was potentially a bond site, which was called "pointpoint-adhesion", and the whole material formed a threedimensional fluffy structure (Fig. 5d-f).

Fig. 6a revealed that the filtration efficiency of calender bonding sample was $92.32 \% \pm 0.5 \%$, which was slightly higher than that of the through-air bonding of $88.61 \% \pm 1.96 \%$. However, the pressure drop of calender bonding sample was $81.34 \pm 4.5 \mathrm{~Pa}$, which was much higher than that of through-air bonding of $22.56 \pm 2.24 \mathrm{~Pa}$. With respect to quality factors (Fig. 6b), we can find out that the filtration performance of through-air bonding sample (quality factor value of $0.096 \pm$ $0.0065 \mathrm{~Pa}^{-1}$ ) was better than calender bonding sample (quality factor value of $0.032 \pm 0.0051 \mathrm{~Pa}^{-1}$ ). It may be resulted from "thin film shape" at the bonding area in calender bonding sample (Fig. 5b), which would obstruct the flow of air, as well as increase the resistance. Additionally, the through-air bonding materials exhibited a high porosity $(P)$ of $95.5 \% \pm 1.5 \%$ (Fig. $6 \mathrm{c}$ ), indicating that the air flow could choose to pass through the media in a shorter and unblocked path, which obeyed the minimal resistance principle. ${ }^{22}$ The porosity was calculated by the following formula:

$$
P=\left(1-\frac{m}{\rho \delta}\right) \times 100 \%
$$

In the formula above, $\rho, m$, and $\delta$ represented the fiber density, $\mathrm{g} \mathrm{m}^{-3}$; web density, $\mathrm{g} \mathrm{m}^{-2}$; and web thickness, $m$. Thus, the through-air bonding materials possessed a smaller pressure drop with higher permeability, which matched very well with the aforementioned filtration performance.

Taking into account the differences in structure and porosity, we conducted a test of the dust holding capacity. Generally, the dust holding capacity, a crucial indicator of the service life of filtration materials, is widely used to evaluate the application performance of fresh air filter. ${ }^{27,28}$ Fig. 6d 

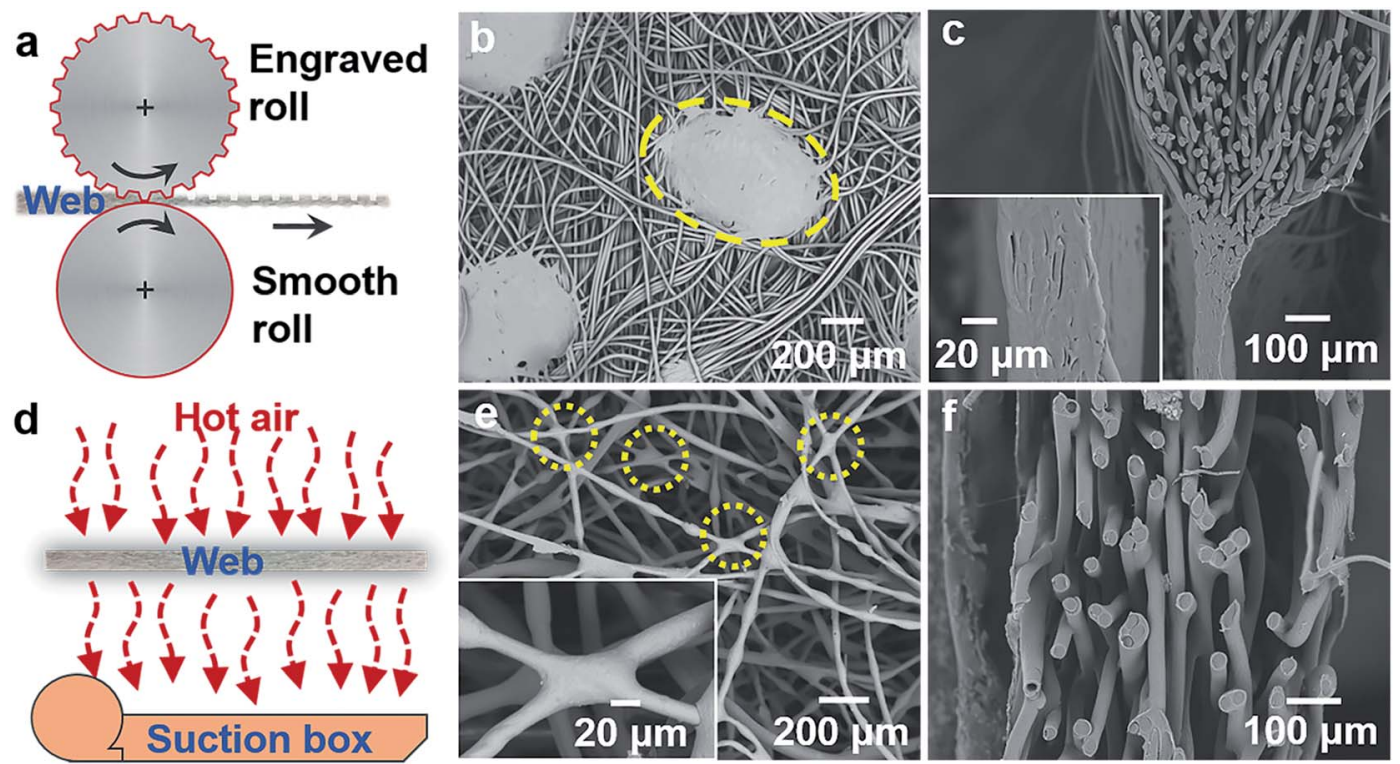

Fig. 5 Schematics diagram of (a) calender bonding process and (d) through-air bonding process. SEM images of calender point-bonding fabric (b) surface morphology, (c) cross section, and through-air bonding fabric (e) surface morphology, (f) cross section.

presented the comparison of the dust holding capacity of calender bonding sample $\left(\sim 5.35 \pm 0.45 \mathrm{~g} \mathrm{~m}^{-2}\right)$ and throughair bonding sample which exhibited significant superiority $\left(\sim 7.23 \pm 0.42 \mathrm{~g} \mathrm{~m}^{-2}\right)$. The above two samples were fabricated with different thermal bonding methods. This result could be explained by the three-dimensional fluffy structure. And in the meanwhile, high porosity extended the time of airborne particles through a filter medium, which significantly increased the collision of particles and the surface of fiber, especially fine particles. ${ }^{21}$

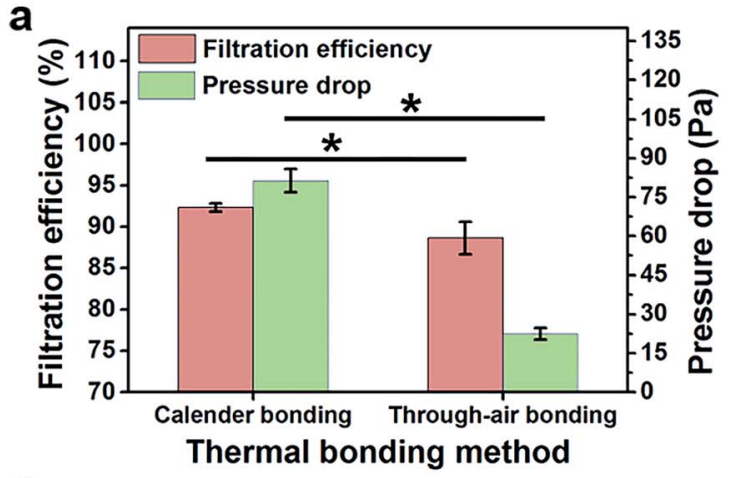

C

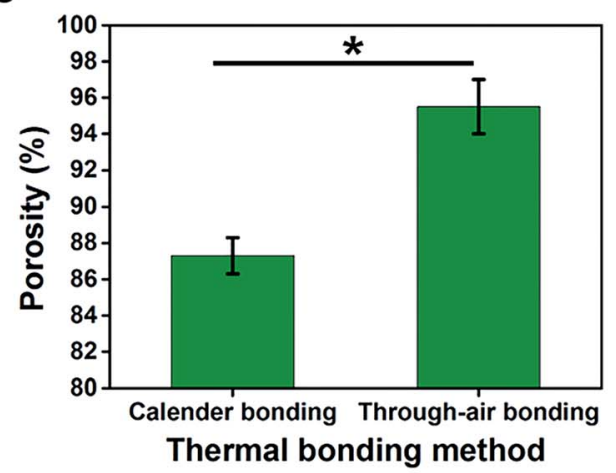

b

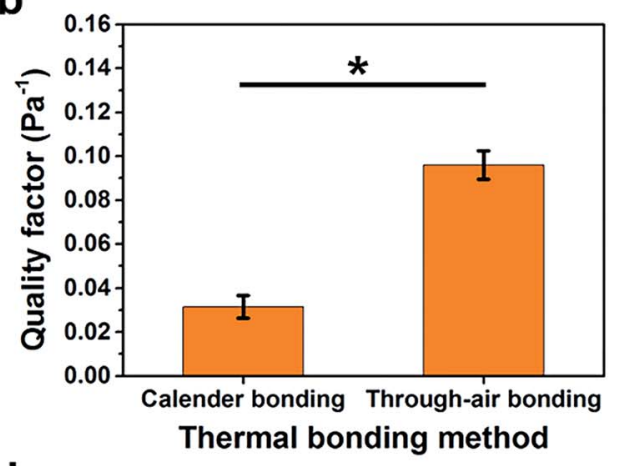

d

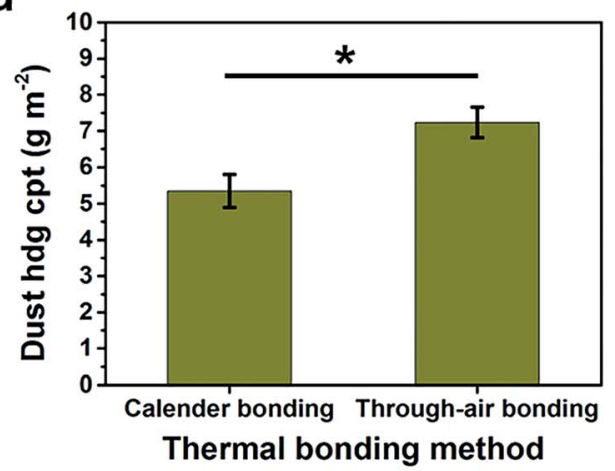

Fig. 6 (a) Filtration performance, (b) quality factor, (c) porosity, and (d) dust holding capacity (abbrev. dust hdg cpt) of thermal bonding samples with different thermal reinforcement methods. 


\section{Evaluation of filtration performance}

Fig. 7a showed the process of $\mathrm{NaCl}$ aerosol particles with mass mean diameter of $0.26 \mu \mathrm{m}$ was filtered by spunbond materials, from which we could clearly observe that $\mathrm{NaCl}$ particles were intercepted on the surface of the fibers and the interior of the materials as well (Fig. 7b-d). The variation regarding the basis weight of the samples could undoubtedly affect the values of the filtration efficiency. The filtration performance of the throughair bonding BCS materials with various basis weights was presented in Fig. 8a. The filtration efficiencies of the BCS materials with basis weight of $50,70,95,120,160$, and $200 \mathrm{~g} \mathrm{~m}^{-2}$ were $59.54 \% \pm 1.73 \%, 70.62 \% \pm 0.97 \%, 83.74 \% \pm 0.65 \%, 88.61 \% \pm$ $1.96 \%, 95.01 \% \pm 1.12 \%$, and $97.02 \% \pm 0.8 \%$, while the pressure drops were $8.45 \pm 0.54,12.59 \pm 1.64,17.85 \pm 3.01,22.62 \pm 2.24$, $27.48 \pm 2.5$, and $35.14 \pm 2.01 \mathrm{~Pa}$, respectively, indicating a synchronous increase upon improving the basis weight. It was clearly seen that the filtration showed a sharp rise and then revealed a relatively high level of value $(\geq 95 \%)$ when the basis weight excessed $160 \mathrm{~g} \mathrm{~m}^{-2}$, while maintaining a relatively low pressure drop of $27.48 \pm 2.5 \mathrm{~Pa}$, which was unachievable for the traditional fresh air filtration materials. The QF of the materials was slightly floating, but basically maintained a desirable value above $0.095 \mathrm{~Pa}^{-1}$ (Fig. 8b). Considering the complex application environment for the air filters, the filtration efficiency and pressure drop as a function of airflow speed $\left(20-100 \mathrm{~L} \mathrm{~min}^{-1}\right)$ were systematically studied to expound the remarkable performances and promising perspectives of the BCS materials with basis weight of $200 \mathrm{~g} \mathrm{~m}^{-2}$ (Fig. 8c). As the airflow speed increased, the filtration efficiency decreased slowly and reached a stable value of $91.3 \% \pm 1.5 \%$ when the airflow was under the highest speed of $100 \mathrm{~L} \mathrm{~min}^{-1}$. In addition, we observed that there was a nearly linear relationship between pressure drop and airflow, which was consistent with Darcy's law for viscous resistance. ${ }^{29}$ The slope of the curve of pressure drop versus air flow velocity was only 0.79 , which was much smaller than the nanoscale ultrafine fiber materials (e.g., 1.49 for $\mathrm{PSU}^{\mathrm{TiO}}{ }_{2}$, 3.67 for PAN/PU), ${ }^{30,31}$ revealing that there was remarkable air permeability in practical applications.

We also carried out the measurement of the dust holding capacity with the purpose of observing the ability of containing dust. Fig. 8d presented the comparison of the dust holding capacity between electrospun materials, melt-blown materials and BCS materials. The dust holding capacity of electrospun materials and melt-blown materials were $0.61 \pm 0.15$ and $3.12 \pm 0.41 \mathrm{~g} \mathrm{~m}^{-2}$, respectively, while BCS materials with basis weight of $200 \mathrm{~g} \mathrm{~m}^{-2}$ achieved a relatively high value of $9.36 \pm 0.52 \mathrm{~g} \mathrm{~m}^{-2}$. This was because that the former two materials mainly intercepted particles on the surface, while the BCS materials could rely on their bulky structure to intercept a good number of particles in the interior, which was in accordance with deep bed filtration deposition patterns (Fig. S4†). To reach the industrial standard of 1000 $\mathrm{Pa}$, it took electrospun materials, melt-blown materials, and BCS materials $9.6 \pm 0.58,17.6 \pm 0.6$, and $32.1 \pm 0.97$ minutes respectively. By comparing the time differences, it was further proved that BCS materials outstood with respect to service life (Fig. $55 \dagger$ ). In addition, we managed to observe that the filtration efficiency of the three materials was comparable, but the pressure drops of through-air bonding PE/PP BCS materials (35.14 \pm $2.01 \mathrm{~Pa}$ ) was much lower than those of electrospun materials $(130.24 \pm 5.39 \mathrm{~Pa})$ and melt-blown materials $(50.6 \pm 1.87 \mathrm{~Pa})$, showing an improved filtration performance in prolonging service life and reducing energy consumption.
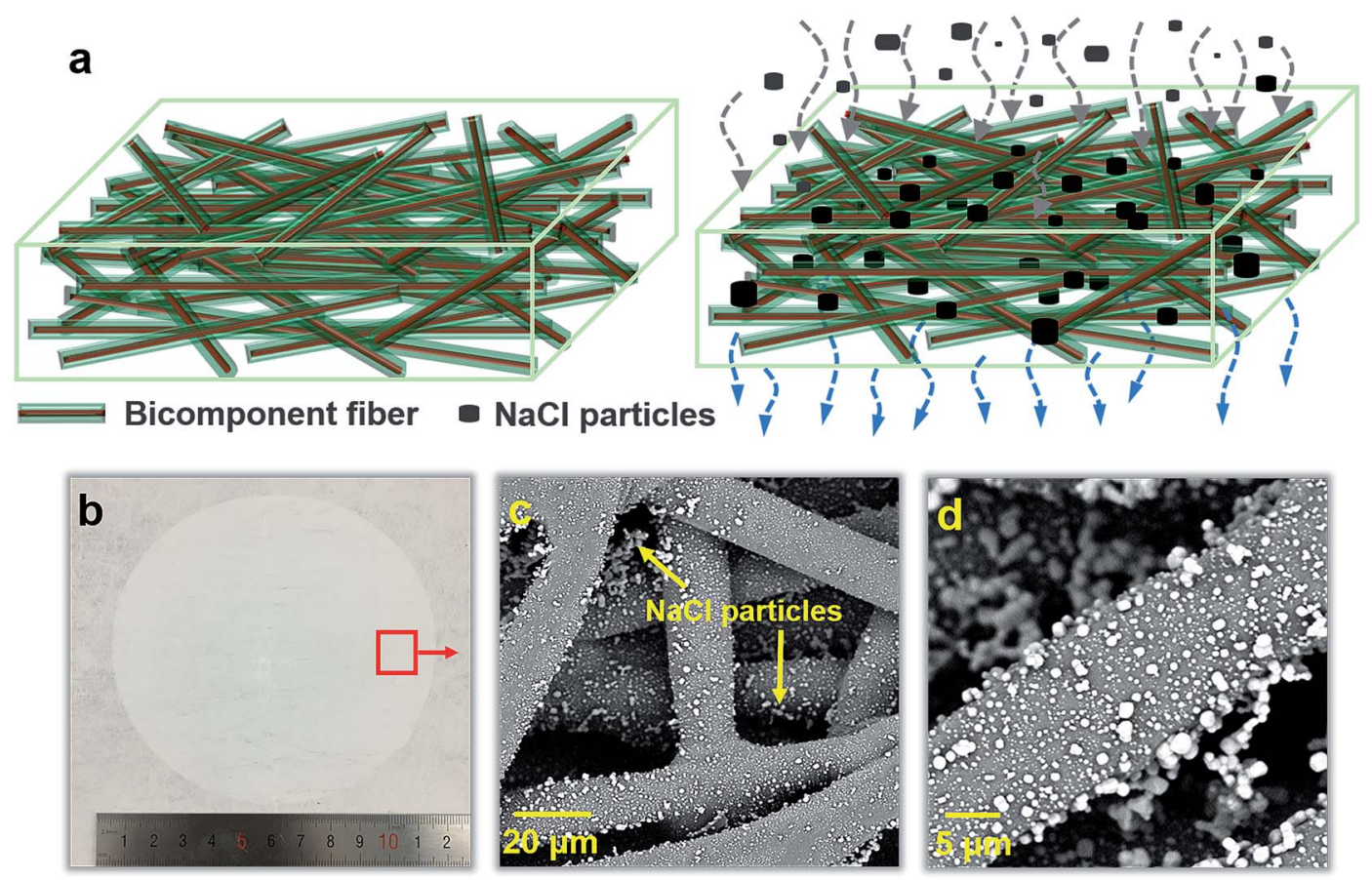

Fig. 7 (a) The filtration process of BCS materials for the mass mean diameter of $0.26 \mu \mathrm{m} \mathrm{NaCl}$ aerosol particles. (b) Photograph and (c, d) SEM images of BCS materials after filtration loading test. 
a

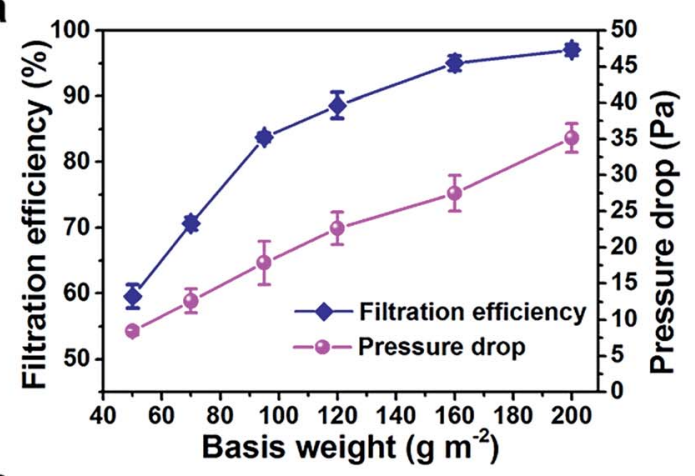

C

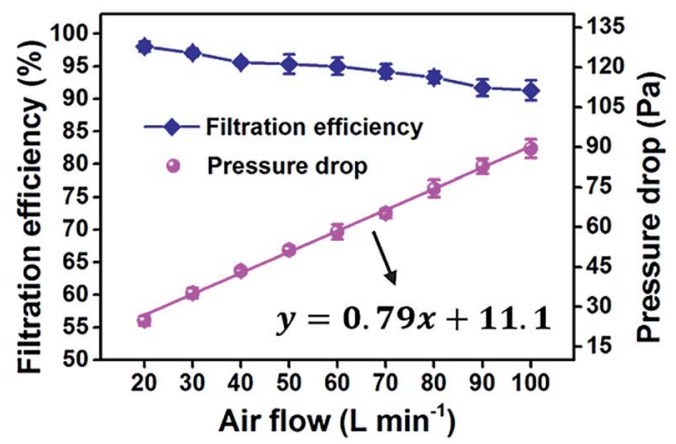

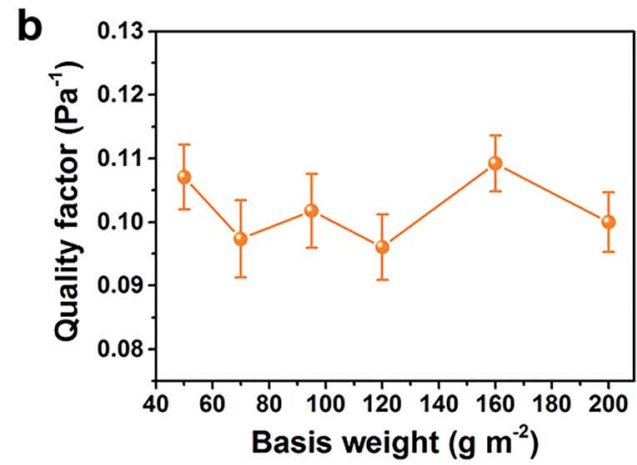

d

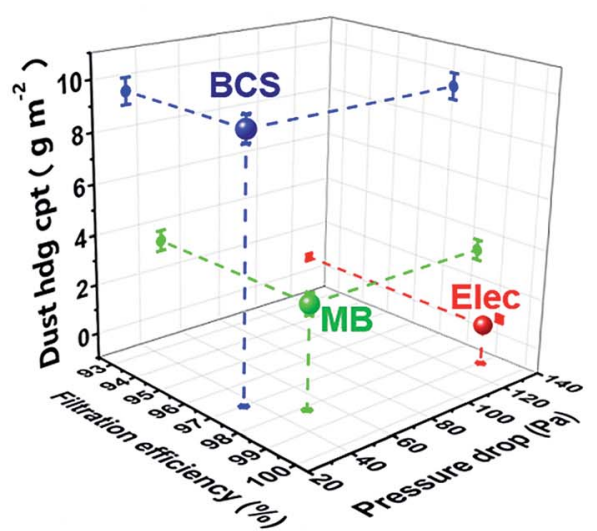

Fig. 8 (a) Filtration efficiency and pressure drop, and (b) quality factor of BCS materials (quenching air temperature of $12^{\circ} \mathrm{C}$, drawing air pressure of $200 \mathrm{kPa}$, through-air bonding) with various basis weights. (c) Filtration efficiency, and pressure drop versus air flow of the BCS materials $\left(\sim 200 \mathrm{~g} \mathrm{~m}^{-2}\right)$. (d) Comparison of the dust holding capacity (abbrev. dust hdg cpt) between electrospun materials (Elec), melt-blown materials (MB) and bicomponent spunbond materials (BCS) under loading pressure drop of $1000 \mathrm{~Pa}$.

\section{Conclusions}

In summary, the PE/PP BCS materials with low resistance and high dust holding capacity for fresh air filtration were successfully fabricated via spunbond technique. By systematically investigating the processing parameters, BCS materials with ideal fluffy and highly porous structure were obtained by using through-air bonding reinforcement when the optimized quenching air temperature and the drawing air pressure were $12{ }^{\circ} \mathrm{C}$ and $200 \mathrm{kPa}$, respectively. The through-air bonding BCS materials with basis weight of $200 \mathrm{~g} \mathrm{~m}^{-2}$ presented a robust filtration performance with a high filtration efficiency of $97.02 \% \pm 0.8 \%$, low pressure drop of $35.14 \pm 2.01 \mathrm{~Pa}$ and excellent dust holding capacity of 9.36 $\pm 0.52 \mathrm{~g} \mathrm{~m}^{-2}$, which could mostly satisfy the requirements of fresh air ventilation system for reducing energy consumption and prolonging service life. The PE/PP BCS materials are expected to have a wider application in clean rooms, engine intake, respirators, as well as medical equipment.

\section{Conflicts of interest}

There are no conflicts to declare.

\section{Acknowledgements}

This work is supported by the Key Technologies R\&D Program of China (No. 2015BAE01B01).

\section{Notes and references}

1 C. Liu, P. C. Hsu, H. W. Lee, M. Ye, G. Zheng, N. Liu, W. Li and Y. Cui, Nat. Commun., 2015, 6, 6205.

2 R. D. Peng, M. L. Bell, A. S. Geyh, A. McDermott, S. L. Zeger, J. M. Samet and F. Dominici, Environ. Health Perspect., 2009, 117, 957-963.

3 S. Feng, D. Gao, F. Liao, F. Zhou and X. Wang, Ecotoxicol. Environ. Saf., 2016, 128, 67-74.

4 Z. Chen, J. N. Wang, G. X. Ma and Y. S. Zhang, Lancet, 2013, 382, 1959-1960.

5 Y. Liu, B. Cheng, N. Wang, W. Kang, W. Zhang, K. Xing and W. Yang, J. Appl. Polym. Sci., 2012, 124, 296-301.

6 Z. Wang, C. Zhao and Z. Pan, J. Colloid Interface Sci., 2015, 441, 121-129.

7 A. Zamani and B. Maini, J. Pet. Sci. Eng., 2009, 69, 71-88.

8 K. W. Lee and B. Y. H. Liu, Aerosol Sci. Technol., 1982, 1, 147161.

9 V. Jegatheesan and S. Vigneswaran, Crit. Rev. Environ. Sci. Technol., 2005, 35, 515-569.

10 J. Liu, D. Y. Pui and J. Wang, Sci. Total Environ., 2011, 409, 4868-4874.

11 R. Thakur, D. Das and A. Das, J. Text. Inst., 2014, 105, 676684.

12 M. Nifuku, Y. Zhou, A. Kisiel, T. Kobayashi and H. Katoh, J. Electrost., 2001, 51, 200-205. 
$13 \mathrm{H}$. Lim, Journal of Textile and Apparel Technology and Management, 2010, 6, 1-13.

14 D. Zhang, C. Sun, J. Beard, H. Brown, I. Carson and C. Hwo, J. Appl. Polym. Sci., 2002, 83, 1280-1287.

15 Y. Li and C. W. Joo, Fibers Polym., 2012, 13, 456-465.

16 Z. Heng, Q. Xiaoming, Z. Qi and Y. Zhaohang, J. Ind. Text., 2014, 45, 48-65.

17 D. Zhang, C. Sun and H. Song, J. Appl. Polym. Sci., 2004, 94, 1218-1226.

18 M. Dasdemir, B. Maze, N. Anantharamaiah and B. Pourdeyhimi, J. Mater. Sci., 2012, 47, 5955-5969.

19 E. Shim, B. Pourdeyhimi and D. Shiffler, J. Appl. Polym. Sci., 2016, 133, 44225.

20 R. Uppal, G. Bhat, C. Eash and K. Akato, Fibers Polym., 2013, 14, 660-668.

21 W. W. F. Leung, C. H. Hung and P. T. Yuen, Sep. Purif. Technol., 2010, 71, 30-37.

22 Y. J. Yang, S. C. Zhang, X. L. Zhao, J. Y. Yu and B. Ding, Sep. Purif. Technol., 2015, 152, 14-22.
23 N. Wang, X. F. Wang, B. Ding, J. Y. Yu and G. Sun, J. Mater. Chem., 2012, 22, 1445-1452.

24 B. Liu, S. Zhang, X. Wang, J. Yu and B. Ding, J. Colloid Interface Sci., 2015, 457, 203-211.

25 C. H. Hung and W. W. F. Leung, Sep. Purif. Technol., 2011, 79, 34-42.

26 R. Nanjundappa and G. S. Bhat, J. Appl. Polym. Sci., 2005, 98, 2355-2364.

27 E. S. Lee, C. C. Fung and Y. Zhu, Environ. Sci. Technol., 2015, 49, 3358-3365.

28 W. J. Fisk, D. Faulkner, J. Palonen and O. Seppanen, Indoor Air, 2002, 12, 223-234.

29 S. Whitaker, Transp. Porous Media, 1986, 1, 3-25.

30 H. Wan, N. Wang, J. Yang, Y. Si, K. Chen, B. Ding, G. Sun, M. El-Newehy, S. S. Al-Deyab and J. Yu, J. Colloid Interface Sci., 2014, 417, 18-26.

31 N. Wang, Z. Zhu, J. Sheng, S. S. Al-Deyab, J. Yu and B. Ding, J. Colloid Interface Sci., 2014, 428, 41-48. 